humans while altering the abundance and distribution of certain disease vectors.

A reigning narrative exists that the consumption of exotic animals represents the sole threat for pandemics to emerge. In reality, the excess consumption of chicken, beef, and pork equally contributes to this problem. To maximize profit from meat production, animals are tightly packed together and specific genes that code for desirable traits (eg, large chicken breasts) are selected. This plethora of genetically identical animals constitutes the perfect environment for a disease to spread rapidly. Additionally, from close confinement to operations done without anesthesia, these animals are subjected to an immense amount of stress that renders them more vulnerable hosts. ${ }^{3}$ Furthermore, to counteract all the aforementioned unhealthy conditions, the routine use of prophylactic antimicrobials has been adopted in factory farms, a fact that greatly exacerbates the existing issue of antimicrobial resistance. ${ }^{7}$ Numerous examples of zoonoses that originated from meat farms already exist, like the outbreak of Nipah virus in Malaysia in the 1990s and the H1N1 pandemic in 2009. Now, more than ever, it has become evident that by abandoning industrialized farming and returning to a smaller model of raising livestock, we can maintain meat production and simultaneously reduce epidemic and pandemic risk and address the matter of animal welfare.

The eliciting factor of civil unrest is not only human overpopulation but also the innate human desire for dominance. Civil unrest can cultivate a breeding ground for an epidemic or a pandemic to erupt. In the case of the Ebola outbreak, social conflicts in the Democratic Republic of the Congo increased human-to-human transmission and devastated the regional healthcare system. ${ }^{8}$ Due to these civil disturbances, there was also a delay in the detection of the epidemic, which was officially declared after it had already spread to neighboring countries. Furthermore, political disorders and civil wars trigger human migration and cohabitation in conditions which are proper for infectious diseases to emerge. ${ }^{9}$ Poor hygiene and sanitation, malnutrition, and difficulties in healthcare supply in refugee camps have caused a lot of epidemic outbreaks during the last decade.

The effects of the anthropogenic alteration of ecosystems along with the continuous pursuit of profit are now indisputably linked to infectious disease emergence. Although we tend to portray ourselves as the victims of pandemics, in truth we are the facilitators. Drastic and decisive measures that address the aforementioned issues on a global level are urgently needed. The sooner we comprehend that "planetary health" and human health are entirely interconnected, the sooner we can start rebuilding the fundamental relationship between us and nature.

Acknowledgments. We thank Mr. Ognyan Iskrenov for assistance in preparing Figure 1.

Financial support. No financial support was provided relevant to this article.

Conflicts of interest. All authors report no conflicts of interest relevant to this article.

\section{References}

1. Taylor LH, Latham SM, Woolhouse ME. Risk factors for human disease emergence. Philos Trans R Soc Lond B Biol Sci 2001;356:983-989.

2. McFarlane R, Sleigh A, McMichael T. Synanthropy of wild mammals as a determinant of emerging infectious diseases in the Asian-Australasian region. Ecohealth 2012;9:24-35.

3. Jones B. Eating meat and not vaccinating: in defense of the analogy. Bioethics 2021;35:135-142.

4. Zhang T, Wu Q, Zhang Z. Probable pangolin origin of SARS-CoV-2 associated with the COVID-19 outbreak. Curr Biol 2020;30:1578.

5. Bezerra-Santos MA, Mendoza-Roldan JA, Thompson RCA, Dantas-Torres F, Otranto D. Illegal wildlife trade: a gateway to zoonotic infectious diseases. Trends Parasitol 2021;37:181-184.

6. Priyadarsini SL, Suresh M, Huisingh D. What can we learn from previous pandemics to reduce the frequency of emerging infectious diseases like COVID-19? Glob Transit 2020;2:202-220.

7. Cheng G, Ning J, Ahmed S, et al. Selection and dissemination of antimicrobial resistance in Agri-food production. Antimicrob Resist Infect Control 2019;8:158.

8. Wells CR, Pandey A, Ndeffo Mbah ML, et al. The exacerbation of Ebola outbreaks by conflict in the Democratic Republic of the Congo. Proc Nat Acad Sci 2019;116:24366-24372.

9. Spernovasilis N, Siakallis G, Tsiodras S, Poulakou G. Child refugees in Europe and infectious diseases: threat or threatened? Int J School Health 2017;4:1-3.

\title{
Tuberculosis behind bars in Latin America and Caribbean: A growing public health crisis
}

\author{
Zarmina Islam ${ }^{1}$, Pawan Kumar Thada², Zainab Syyeda Rahmat ${ }^{1}$, Samaa Akhtar ${ }^{3}$, Shkaib Ahmad ${ }^{4}$, \\ Ana Carla dos Santos Costa ${ }^{5}$, Mohammad Mehedi Hasan ${ }^{6,7}$, Shoaib Ahmad ${ }^{2}$ and Mohammad Yasir Essar ${ }^{8}$ \\ ${ }^{1}$ Dow University of Health Sciences, Karachi, Pakistan, ${ }^{2}$ Punjab Medical College, Faisalabad, Pakistan, ${ }^{3}$ University of London, London, United Kingdom, ${ }^{4}$ Dera \\ Ghazi Khan Medical College, Punjab, Pakistan, ${ }^{5}$ Faculty of Medicine, Federal University of Bahia, Salvador, Bahia, Brazil, ${ }^{6}$ Department of Biochemistry and \\ Molecular Biology, Faculty of Life Science, Mawlana Bhashani Science and Technology University, Tangail, Bangladesh, ${ }^{7}$ Division of Infectious Diseases, \\ The Red-Green Research Centre, BICCB, Dhaka, Bangladesh and ${ }^{8}$ Kabul University of Medical Sciences, Kabul, Afghanistan
}

Author for correspondence: Mohammad Yasir Essar, E-mail: yasir.essar@gmail.com Cite this article: Islam Z, et al. (2022). Tuberculosis behind bars in Latin America and Caribbean: A growing public health crisis. Infection Control \& Hospital Epidemiology, 43: 2000-2002, https://doi.org/10.1017/ice.2021.424
To the Editor-Despite advances in recent years to end the tuberculosis (TB) epidemic and global efforts to reduce its prevalence, the disease remains a burden on public health in several countries. In Latin America and the Caribbean, the situation is no different. With incidence rates of 61.2 per 100,000 population in the Caribbean, 46.2 in South America, and 25.9 in Mexico and 
Central America, the region is at risk of falling short of the "End TB Strategy" goals. ${ }^{1}$

TB transmission correlates with susceptible populations, which include the socioeconomically disadvantaged, migrants, ethnic minorities, individuals with comorbidities (eg, human immunodeficiency virus [HIV]), and persons deprived of their liberty (PDL). According to the World Health Organization (WHO), the incidence rate of TB is 100 times greater among inmates compared to the general population, and $\mathrm{TB}$ in $\mathrm{PDL}$ continues to increase because of gross neglect. ${ }^{1}$

The disparity between prison and civilian TB control measures and the lack of policies to safeguard prison healthcare are the primary concerns. Overcrowding in prisons of Central and South America contributed to a $206 \%$ average increase in TB since 2000 , higher than in any region. PDL TB in this region increased from $5 \%$ of the total reported cases in 2011 to $11.1 \%$ in $2017 .^{2}$ El Salvador, for example, had the greatest increase of $411 \%$ among TB PDL, with a corresponding increase in TB of $51.5 \%$, in 2017.3 In Brazil, the increase in the incarceration rate has led to more PDL than capacity in detention centers, with average occupancy at $161 \%$ of availability. A similar trend has been noted in other American countries. ${ }^{4}$

Both the prison environment and host factors are responsible for TB surges. Globally, prisons have limited access to healthcare and medical facilities, lack of well-trained medical staff, an insufficient supply of newly updated quality medicine regimen, poor ventilation and sanitation, deficient new and upgraded diagnostic approach and laboratories, and malnutrition. These factors tend to be more conspicuous in Latin America. ${ }^{5,6}$ Additionally, PDL are highly associated with low socioeconomic backgrounds, limited access to health care before incarceration, underprivileged ethnic groups, high tendency of substance misuse, smoking, and sexual abuse once imprisoned, all of which combine to create a nidus for TB and its transmission. ${ }^{4,7}$

Higher evidence of drug trafficking and a small fraction of Directly Observed Treatment Short-Course (DOTS) implementation and completion among PDL in Latin America caused a surge in TB transmission. A recent study showed a lower prevalence of latent TB infection than active infection in Brazil at the time of entry, likely due to high risk thereafter in the prison setting. ${ }^{8}$ Long duration and previous history of incarceration have been identified as risk factors for latent TB infection. Furthermore, incarcerated people are deprived of their human rights-they are socially, emotionally, and psychologically isolated. Access to social welfare organizations, human rights activists, community representatives, and rehabilitation services to support this vulnerable populations is lacking. ${ }^{8}$

Another matter of concern is the increase in multidrug-resistant (MDR)-TB cases. TB screening systems before incarceration are highly neglected, and screening is impeded by logistic obstacles and very short periods of stay for many inmates. ${ }^{7}$ Late diagnoses only following symptoms, inadequate observation of treatment, and monitoring of the disease condition led to the development of MDR-TB. This factor is particularly worrying in the region because $70 \%$ of global RR/MDR-TB cases are concentrated in Central and South America. ${ }^{1}$ In Central and South America, where $77.5 \%$ of TB patients can be treated successfully, mortality among PDL is high and preventable. Among the reasons for these excess deaths among PDL is the nonadherence rate, which is high due to negligence regarding patient education and/or counseling to improve drug adherence.

Additionally, the HIV coinfection rate is high in Central America and Caribbean countries, and TB is the leading infectious cause of death for HIV patients. HIV coinfection also increases
MDR-TB. The high rate of prison turnover, reincarceration, and movement in prisons are less-prioritized factors of TB expansion. Overlapping periods of stay between infected and healthy PDL as well as inevitable contact with guards and prison employees have raised the risk of transmission. Treatment discontinuity is also high due to the circulation of inmates, which has further exacerbated TB spread. ${ }^{7,9}$ Reduced physical activity also makes PDL vulnerable to diseases.

Escalating infectious diseases, use of immunosuppressives, and increases in comorbidities have also made the PDL more susceptible to TB. ${ }^{10,11}$ A cross-sectional study of Brazilian PDL demonstrated that coinfection with viral hepatitis had a higher risk of hepatotoxicity related to the cessation of first-line antituberculosis drugs. ${ }^{10}$ Notably, depending upon the facility type for incarceration, the rate of TB varies. It is estimated to be higher in local jails than in state and federal prisons because of the high population, movement, and turnover in local jails. ${ }^{6}$

The implications of an increase in TB in prisons in Central and South America include spread of TB to visitors, family members, and prison workers. Moreover, admitting PDL to community hospitals and releasing PDL with a history of TB without proper liaison between the prison health system and local health authorities are also contributing to TB circulation to the general public. These factors create a favorable situation for TB spread outside of prisons, potentially to those at high risk, particularly HIV patients.

PDL are not easily accessible to visiting authorities due to legal concerns. Research gaps, lack of funding, and outdated policies to ameliorate prison health services plague most of South and Central America. Increased violence and crime are also major concerns in the TB crisis behind bars. ${ }^{5}$ With the lack of data from these jails, proper implementation cannot take place to prevent TB spread. This situation presents challenges for healthcare systems and frontline workers, for those of low socioeconomic background who cannot afford medical treatment for TB, and for legal systems that need to take precautions to decrease incarceration rates. Implementable actions include, but are not limited to, health and sex education to reduce HIV prevalence and consequently TB, provision of basic healthcare services to local and federal jails, active routine testing of TB in jails, legal interventions to decrease incarceration rates, lower mobility of inmates, educational resources on drug adherence, and routine updates on the development of TB in jails. ${ }^{3,6}$

The increasing incidence of tuberculosis among the PDL of Latin America and the Caribbean can be attributed to several preventable causes: overcrowding in prisons, lack of basic healthcare in prisons, and increased susceptibility to TB due to underlying medical conditions (eg, AIDS). If inmates are not already carriers of TB when incarcerated, overcrowding of prisons increases the chances of acquiring TB while incarcerated. The disproportionate incarceration of poor people and minority groups who may have been marginalized by healthcare systems before incarceration comprises a major risk factor. ${ }^{12}$

A notable limitation of our study was the lack of routinely updated data on the development of TB in jails of Central and South America. Although some viable research articles have been published regarding the TB crisis among PDL of Central and South America, not enough attention has been given to this grave yet preventable crisis. Urgent measures are needed to deal with this growing public health crisis.

\section{Acknowledgments.}

Financial support. No financial support was provided relevant to this article. 
Conflicts of interest. All authors report no conflicts of interest relevant to this article.

\section{References}

1. Tuberculosis in the Americas 2018. World Health Organization website. https://iris.paho.org/bitstream/handle/10665.2/49510/

PAHOCDE18036_eng? sequence $=1$ \&isAllowed $=y$. Accessed September 27, 2021.

2. Pelly T, Moore DAJ, Gilman R, Evans C. Recent tuberculosis advances in Latin America. Curr Opin Infect Dis 2004;17:397.

3. O'Grady J, Maeurer M, Atun R, et al. Tuberculosis in prisons: anatomy of global neglect. Eur Respir J 2011;38:752-754.

4. TB in prisons. World Health Organization website. https://www.who.int/tb/ areas-of-work/population-groups/prisons-facts/en/. Accessed September 27, 2021.

5. Dara M, Acosta CD, Vinkeles Melchers NVS, et al. Tuberculosis control in prisons: current situation and research gaps. Int J Infect Dis. 2015;32:111117. doi: 10.1016/J.IJID.2014.12.029
6. Walter KS, Martinez L, Arakaki-Sanchez D, et al. The escalating tuberculosis crisis in central and South American prisons. Lancet 2021;397:1591-1596.

7. Jones TF, Craig AS, Valway SE, Woodley CL, Schaffner W. Transmission of tuberculosis in a jail. Ann Intern Med 1999;131:557-563.

8. Carbone ASS, Paião DSG, Sgarbi RVE, et al. Active and latent tuberculosis in Brazilian correctional facilities: a cross-sectional study. BMC Infect Dis 2015;15:24.

9. Estevan AO, do Valle Leone de Oliveira SM, Croda J. Active and latent tuberculosis in prisoners in the Central-West Region of Brazil. Rev Soc Bras Med Trop 2013;46:515-518.

10. Puga MAM, Bandeira LM, Pompilio MA, et al. Screening for HBV, HCV, HIV and syphilis infections among bacteriologically confirmed tuberculosis prisoners: an urgent action required. PLoS One 2019;14(8):e0221265.

11. Costa AC dos S, Hasan MM, Xenophontos E, et al. COVID-19 and Zika: an emerging dilemma for Brazil. J Med Virol. 2021;93:4124-4126.

12. New HIV infections rising in Latin America_- key populations particularly affected. United Nations AIDS website. https://www.unaids.org/en/ resources/presscentre/featurestories/2019/october/20191014_latin-america. Accessed July 20, 2021.

\title{
A case of severe acute respiratory coronavirus virus 2 (SARS-CoV-2) reinfection within ninety days of primary infection in a healthcare worker
}

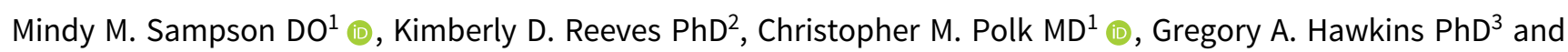 \\ Catherine L. Passaretti MD ${ }^{1}$ (1) \\ ${ }^{1}$ Division of Infection Diseases, Department of Medicine, Atrium Health, Charlotte, North Carolina, ${ }^{2}$ Department of Internal Medicine, Wake Forest School of \\ Medicine, Winston-Salem, North Carolina and ${ }^{3}$ Department of Biochemistry, Wake Forest School of Medicine, Winston-Salem, North Carolina
}

To the Editor-Case definitions and prior literature typically describe reinfections with severe acute respiratory coronavirus virus 2 (SARS$\mathrm{CoV}-2$ ) in situations when someone becomes infected $>90$ days from their prior infection. ${ }^{1}$ Healthcare workers (HCWs) may be particularly susceptible to reinfection. ${ }^{2}$ With the emergence of the o (omicron) variant of SARS-CoV-2, an increased risk of reinfection has been described. ${ }^{3}$ Here, we describe a case of reinfection that occurred only 2 weeks after the primary infection.

A 38-year-old female HCW developed a primary infection with SARS-CoV-2 in November 2021. She had no comorbidities or immunocompromising conditions. She was not vaccinated for SARS-CoV-2. Symptoms at that time consisted of fever, myalgias, headache, fatigue, congestion, and cough. A polymerase chain reaction (PCR) analysis of a nasopharyngeal (NP) swab sample collected 4 days after symptom presentation resulted in cycle threshold $(\mathrm{Ct})$ values of 23.2 and 23.6, respectively, for the $O R F 1$ and $E$ genes on the Roche cobas platform (Roche Diagnostics, Basel, Switzerland). Subsequent analysis of the NP swab sample identified the $\delta$ (delta) variant (sublineage AY.3) as the causal pathogen.

Author for correspondence: Mindy M. Sampson, E-mail: mindy.sampson@ atriumhealth.org

Cite this article: Sampson MM, et al. (2022). A case of severe acute respiratory coronavirus virus 2 (SARS-CoV-2) reinfection within ninety days of primary infection in a healthcare worker. Infection Control \& Hospital Epidemiology, 43: 2002-2003, https://doi.org/10.1017/ice.2022.42
The patient had complete resolution of symptoms. She received 1 dose of dexamethasone during her primary infection but otherwise received no treatment. Then, 7 weeks after her primary diagnosis, she developed fever, sore throat, fatigue, congestion, and cough. An NP swab sample was collected and tested 2 days after symptom onset, which was again positive for SARS-CoV-2. The Ct values were 20.92 and 20.95 for the $O R F 1$ and $E$ genes, respectively, on the Roche cobas platform. The sequence analysis of this second NP swab sample revealed the presence of the o variant (omicron sublineage BA.1), confirming a new infection of SARS-CoV-2.

This is a unique case demonstrating an early reinfection with SARS-CoV-2 occurring within 90 days of prior infection. Reinfection is likely to be more frequent with the o (omicron) lineage due to the escape of this variant from prior neutralizing SARS-CoV-2 antibodies. ${ }^{4,5}$ Importantly, symptomatic individuals should be tested and evaluated for the possibility of reinfection, even within 90 days of their last infection. Understanding the dynamics of reinfections is particularly important as we update testing protocols and infection prevention policies during this surge of the omicron variant, and as we prepare for future emerging variants. This individual did have low Ct values, which would likely represent the presence of live virus; therefore, isolation would have been important to prevent continued spread. ${ }^{6,7}$ This finding also highlights the importance of vaccination in our healthcare workers, given we now know that individuals who have completed their vaccine series and have received a booster are less likely to become infected with the o (omicron) variant. ${ }^{8}$ Cases like 